\title{
Comparaison de coefficients de sécurité pour les pieux sous charges axiales
}

\section{R. FRANK}

CERMES (ENPC-LCPC), Cité Descartes, Champs-sur-Marne
On décrit les divers coefficients de sécurité pour le dimensionnement des pieux sous charges axiales aux états limites ultimes proposés par la prénorme

Eurocode 7 - Partie 1 (CEN, 1994 et AFNOR, 1996) et par les règles du Fascicule $n^{\circ} 62$ - Titre $V$ du ministère français de l'Equipement (MELT, 1993), Les sécurités globales données par les deux codes sont comparées, tant dans le cas où la capacité portante ultime est déduite d'essais exécutés sur le sol (règles pressiométriques) que dans le cas où elle est déduite d'essais de chargement de pieux. Les résultats montrent une bonne concordance. Enfin, une proposition est faite pour remplacer les cas B et $C$ (pour les combinaisons fondamentales) de

l'Eurocode 7-1 par un cas unique, qu'il est aisé d'établir pour le problème de la capacité portante des pieux sous charges axiales.

\section{Comparison of safety coefficients for axially loaded piles}




\section{Introduction}

Pour dimensionner les pieux sous charges axiales en ce qui concerne la capacité portante ultime, la prénorme européenne Eurocode 7 - Partie 1 (CEN, 1994 et AFNOR, 1996) contient tous les coefficients de sécurité à appliquer lorsque des règles de calcul à partir d'essais de sol sont disponibles ou lorsque des essais de chargement statique de pieux sont exécutés. Ce document décrit comment passer de la (ou des) valeur(s) mesurée(s) de la capacité portante ultime à la valeur caractéristique et, de la valeur caractéristique, à la valeur de calcul. Les coefficients partiels à appliquer aux actions (charges) aux états limites ultimes (ELU) sont également connus.

Dans les règlements de calcul de la plupart des pays, on donne également le cheminement entier à suivre pour dimensionner les pieux vis-à-vis des charges axiales.

Ainsi, il est possible, pour le problème de la capacité portante axiale ultime, de comparer la sécurité globale apportée par les Eurocodes (dans leur version provisoire actuelle) à celle préconisée par les pratiques nationales.

Dans le présent article, les comparaisons sont faites avec le règlement officiel français Fascicule $n^{\circ} 62$ - Titre V (MELT, 1993). Pour simplifier, seuls les pieux en compression sont examinés et aucun effet de groupe n'est pris en compte. Par ailleurs, il faut garder présent à l'esprit que ces comparaisons ne concernent pas la sécurité globale finale de la fondation sur pieux, car d'autres éléments interviennent que la capacité portante axiale ultime. Citons, par exemple, le comportement axial aux états limites de service (EI.S), qui est d'ailleurs traité très différemment dans les deux documents.

\section{2}

\section{Relations de base}

Les relations de base de l'Eurocode 7-1 pour la capacité portante ultime sont les suivantes.

$$
\begin{gathered}
\mathrm{R}_{\mathrm{ck}}=\mathrm{R}_{\mathrm{ccm}} / \xi \\
\mathrm{R}_{\mathrm{c}}=\mathrm{R}_{\mathrm{ck}} / \gamma_{\mathrm{s}} \\
\mathrm{R}_{\mathrm{c}}=\mathrm{R}_{\mathrm{bjk}} / \gamma_{\mathrm{b}}+\mathrm{R}_{\mathrm{sk}} / \gamma_{\mathrm{s}}
\end{gathered}
$$

où : $R_{c m}$ est la valeur mesurée de la capacité portante ultime,

$R_{c k}$ est la valeur caractéristique de la capacité portante ultime,

et $\quad R_{c}$ est la valeur de calcul de la capacité portante ultime,

avec: $R_{b k}$ la valeur caractéristique de la résistance de la pointe,

et $\quad R_{s k}$ la valeur caractéristique de la résistance du fût,

$\xi$ les facteurs à appliquer aux valeurs mesurées,

$\gamma_{\text {, }}$ ou $\gamma_{t}$ et $\gamma_{t}$ les coefficients à appliquer à la capacité portante caractéristique totale, ou aux résistances caractéristiques de pointe et de fût, respectivement.
Par ailleurs, les charges appliquées caractéristiques F sont multipliées par les coefficients $\gamma_{F}$ de manière à obtenir la charge de calcul en compression :

$$
\mathrm{F}_{\mathrm{c}}=\gamma_{\mathrm{F}} \mathrm{F}
$$

La condition de base à remplir pour tous les états limites ultimes (ULS) est :

$$
\mathrm{F}_{\text {e(ULS) }} \leq \mathrm{R}_{\mathrm{c}}
$$

Les relations 1 à 4 conduisent à :

$$
\mathrm{F} \leq \mathrm{R}_{\mathrm{cm}} / \gamma_{\mathrm{F}} \cdot \gamma_{1} \cdot \xi=\mathrm{R}_{\mathrm{cm}} / \mathrm{FS}
$$

où $\mathrm{FS}=\gamma_{\mathrm{F}}, \gamma_{1} . \bar{\xi}$ peut être considéré comme le coefficient de sécurité globale. Il faut remarquer que dans la relation (5) $\gamma_{F}$ synthétise, éventuellement, des coefficients sur les charges différents (suivant la nature des charges, voir tableau I, ci-après) et que $\gamma_{\text {r }}$ synthétise $\gamma_{\text {t }}$ et $\gamma$, dans le cas où des coefficients différents sont utilisés pour la pointe et pour le fût.

\section{3}

\section{Valeurs des coefficients $\gamma$}

\section{1}

\section{Eurocode 7-1}

Pour l'Eurocode 7-1, pour les ELU fondamentaux (situations durables et transitoires), les valeurs de $\gamma_{\mathrm{F}}$ pour les cas B et $C$ et de $\gamma_{t}$ ou de $\gamma_{b}$ et $\gamma_{s}$, pour le cas $C$,

\begin{tabular}{|c|c|c|c|}
\hline \multirow[b]{3}{*}{ Cas } & \multicolumn{3}{|c|}{ Actions } \\
\hline & \multicolumn{2}{|c|}{ Permanentes } & Variables \\
\hline & défavorables & favorables & défavorables \\
\hline B & {$[1,35]$} & {$[1,00]$} & {$[1,50]$} \\
\hline C & {$[1,00]$} & {$[1,00]$} & {$[1,30]$} \\
\hline
\end{tabular}
sont donnés dans les tableaux I et II, respectivement. Pour le cas B, $\gamma_{1}$, ou $\gamma_{b}$ et $\gamma_{5}=[1,00]$. Dans les situations accidentelles tous ces coefficients sont égaux à $[1,00]$.

TABleaul Eurocode 7-1: Coefficients partiels $\gamma_{\mathrm{F}}$. Situations ELU fondamentales.

TABLEAU II Eurocode 7-1: Coefficients partiels $\gamma_{b}, \gamma_{s}$ et $\gamma_{t}$. Situations ELU fondamentales, cas C.

\begin{tabular}{l|c|c|c}
\hline Facteurs & $\gamma_{i}$ & $\gamma_{i}$ & $\gamma_{i}$ \\
\hline Pieux foncés & {$[1,3]$} & {$[1,3]$} & {$[1,3]$} \\
\hline Pieux forés & {$[1,6]$} & {$[1,3]$} & {$[1,5]$} \\
\hline $\begin{array}{l}\text { Pieux CFA } \\
\text { (tarière continue) }\end{array}$ & {$[1,45]$} & {$[1,3]$} & {$[1,4]$} \\
\hline
\end{tabular}

Noter que, pour le cas B, $\gamma_{1,} \gamma$, et $\gamma_{1}=(1,00)$.

Remarque: Toutes les valeurs numériques dans les tableaux I et II de la prénorme Eurocode 7-1 sont des valeurs dites " encadrées ", identifiées par []. Cela signifie que ce sont des valeurs indicatives et qu'il appartient aux pays (les «États membres ») du Comité européen de normalisation, CEN) de les fixer. Le Document d'application nationale (DAN), inclus dans la version de la prénorme Eurorode 7-1 éditée par l'AFNOR (1996), préconise d'appliquer en 
France les valeurs numériques encadrées (c'est-àdire de conserver les valeurs numériques indicatives proposées).

\section{2}

\section{Fascicule 62-V}

Pour les états limites ultimes fondamentaux (situations durables et transitoires), les coefficients partiels $\gamma_{F}$ sur les charges du Fascicule $n^{\circ} 62$ - Titre V sont très semblables à ceux du cas $\mathrm{B}$ de l'Eurocode 7 . En particulier, ce coefficient vaut 1,35 pour les charges permanentes défavorables, il vaut 1,00 sur les charges permanentes favorables et 1,5 pour les actions variables de base (sauf en ce qui concerne les charges d'exploitation étroitement bornées ou de caractère particulier). La combinaison des charges pour les situations accidentelles est la même que celle de l'Eurocode 7 (pour plus de détails, on pourra se reporter à Frank, 1994).

Dans le Fascicule $n^{\circ} 62$ - Titre V, on utilise la même valeur $\gamma$ pour la résistance de pointe et pour la résistance de fût. Cette valeur est :

$$
\gamma_{\mathrm{b}}=\gamma_{\mathrm{s}}=\gamma_{\mathrm{s}}=1,4
$$

pour les combinaisons ELU fondamentales;

$$
\gamma_{\mathrm{b}}=\gamma_{\mathrm{s}}=\gamma_{\mathrm{t}}=1,2
$$

pour les combinaisons ELU accidentelles.

\section{4}

\section{Estimation des valeurs caractéristiques de la capacité portante}

On doit distinguer deux cas (voir paragraphe 7.6.3 de l'Eurocode 7-1):

1) la justification est effectuée d'après les résultats d'essais exécutés sur le sol ;

2) la justification est effectuée d'après les résultats d'essais de chargement de pieux.

\section{1}

\section{Calcul sur la base d'essais exécutés sur le sol}

Quand les résultats d'essais de sol sont utilisés, la clause (4)P du paragraphe 7,6.3.3 de l'Eurocode 7-1 stipule que :

« Les valeurs caractéristiques de $\mathrm{q}_{\mathrm{bx}}$ et $\mathrm{q}_{\mathrm{sik}}$ doivent être déduites au moyen de règles de calcul fondées sur des corrélations établies entre les résultats d'essais de chargement statique et les résultats d'essais in situ, ou en laboratoire, sur le terrain. Ces règles doivent être conçues de telle sorte que les capacités portantes ultimes obtenues en utilisant les valeurs caractéristiques $q_{b k}$ et $q_{s i k}$ ne dépassent pas les capacités portantes ultimes mesurées, utilisées pour établir les corrélations divisées par [1,5] en moyenne. 1) $\left(\mathrm{q}_{\mathrm{bk}}\right.$ est la valeur caractéristique de la résistance par unité de surface de la base du pieu et $q_{\text {sik }}$ est la valeur caractéristique par unité de surface du fût du pieu dans la couchei.)

En d'autres termes, $\xi$ doit être «en moyenne » égal à $1,5: \xi_{\text {uve }}=1,5$.
Le Fascicule $n^{\circ} 62$ - Titre $V$ donne deux méthodes de calcul à partir de résultats d'essais in situ : une méthode à partir des essais au pressiomètre Ménard (MPT) et une méthode à partir des essais de pénétration statique (CPT) ${ }^{(1)}$. Ces deux méthodes proviennent de corrélations avec de nombreux essais de chargement statique de pieux (développées, à l'origine, par Bustamante et Gianeselli, 1981). Il faut également relever que les essais statiques de pieux sont exécutés en France d'une manière tout à fait conforme à celle de 1'ISSMFE (1985) recommandée par l'Eurocode 7-1.

Afin de pouvoir comparer à l'Eurocode 7-1, le problème central pour toutes les méthodes ou règles de calcul est de connaître la valeur de $\xi_{\text {ave. }}$. Pour les règles pressiométriques contenues dans le Fascicule $n^{\circ} 62$ Titre $V$, une première estimation a été effectuée sur un nombre limité de résultats d'essais de chargement par J. Renault au SETRA, en 1996. Cette estimation mène à $\xi_{\text {ave }}=1,25$ pour l'ensemble des pieux (pieux foncés et pieux battus confondus). Elle est représentée sur la figure 1.

\section{2}

\section{Calcul sur la base d'essais de chargement statique de pieux}

Les valeurs de $\xi$ proposées par l'Eurocode 7-1 sont données par le tableau III. On doit retenir la plus petite des deux valeurs de capacité portante obtenues par l'application des conditions a) et b).

Dans le cas du Fascicule $n^{\circ} 62$ - Titre V: tué:

- si un seul essai de chargement de pieu est effec-

$$
\xi=1,2
$$

- si plusieurs essais de chargement sont effectués, la valeur de la capacité portante ultime à retenir est déterminée directement à partir d'une évaluation critique des résultats des essais. Cependant, lorsque la

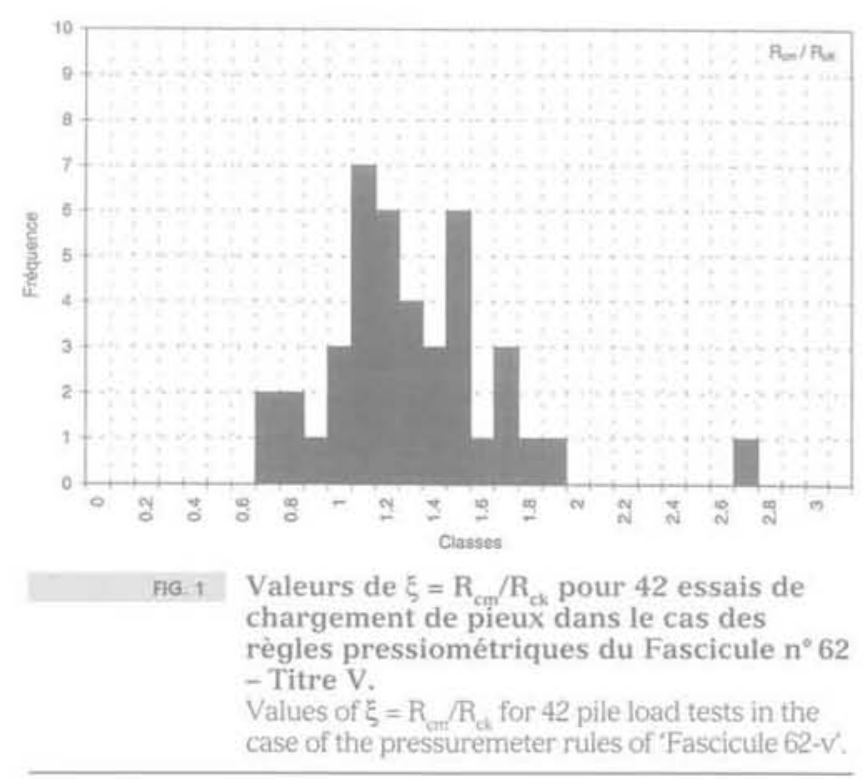

(17 Le détail de ces deux méthodes du Fascicule $n^{\circ} 62$ - Titre $V$ est donné, en langue anglaise, dans le rapport national pour la France présenté au récent séminaire international de l'ERTC3 à Bruxelles (Bustamante et Frank. 1997). 
dispersion n'atteint pas $30 \%$, on peut utiliser la relation suivante (et c'est ce que nous ferons par la suite) :

$$
\mathrm{R}_{\mathrm{ck}}=\mathrm{R}_{\min }\left(\mathrm{R}_{\min } / \mathrm{R}_{\max }\right) \xi^{\prime}
$$

où $R_{\text {min }}$ et $R_{\text {max }}$ sont les valeurs minimale et maximale mesurées et $\xi$ est donné dans le tableau IV.

\section{TARIEAUIII Eurocode 7-1: Facteurs $\xi$ pour le calcul de $R_{c k}$.}

\begin{tabular}{l|c|c|c}
$\begin{array}{l}\text { Nombre d'essais } \\
\text { de chargement }\end{array}$ & 1 & 2 & $>2$ \\
$\begin{array}{l}\text { a) Facteur } \xi \text { sur } \\
\text { la moyenne de } \mathrm{R}_{\mathrm{cm}}\end{array}$ & {$[1,5]$} & {$[1,35]$} & {$[1,3]$} \\
\hline $\begin{array}{l}\text { b) Facteur } \xi \text { sur la plus } \\
\text { petite valeur de } \mathrm{R}_{\mathrm{cm}}\end{array}$ & {$[1,5]$} & {$[1,25]$} & {$[1,1]$} \\
\hline
\end{tabular}

TABLEAUIV Fascicule $n^{\circ} 62$ - Titre V: Facteurs है'.

\begin{tabular}{l|c|c|c|c}
\hline $\begin{array}{l}\text { Nombre diessais } \\
\text { de chargement }\end{array}$ & 2 & 3 & 4 & 5 \\
\hline$\xi^{\prime}$ & 0,55 & 0,20 & 0,07 & 0,00 \\
\hline
\end{tabular}

\section{5}

\section{Comparaisons de la sécurité globale}

Dans ce qui suit, les valeurs de FS $=\gamma_{\mathrm{F}} \cdot \gamma_{1} \cdot \xi$ (équation 5) sont déterminées dans le cas de l'Eurocode 7-1 et dans le cas du Fascicule $n^{\circ} 62$ - Titre V. Pour les ELU fondamentaux, toutes les actions sont considérées comme défavorables.

\section{1}

\section{Calcul sur la base d'essais pressiométriques}

Dans le cas du Fascicule $n^{\circ} 62$ - Titre V, on suppose que les règles pressiométriques sont utilisées et que $\xi$ ave $=1,25$. Pour l'Eurocode $7-1$, on utilise ici les mêmes coefficients $\gamma_{1}$ pour les résistances de pointe et de fût, comme c'est le cas dans le Fascicule $n^{\circ} 62$ - Titre V.

\section{- COMBINAISONS FONDAMENTALES}

\section{Eurocode 7-1}

Cas B : $100 \%$ de charges permanentes :

$\mathrm{FS}=1,35 \times 1,0 \times 1,5=2,03$

$67 \%$ de charges permanentes et $33 \%$ de charges variables :

$\mathrm{FS}=1,4 \times 1,0 \times 1,5=2,10$

$50 \%$ de charges permanentes et $50 \%$ de charges variables :

$\mathrm{FS}=1,425 \times 1,0 \times 1,5=2,14$

Cas C :

pieux foncés, $100 \%$ de charges permanentes :

$\mathrm{FS}=1,0 \times 1,3 \times 1,5=1,95$

$67 \%$ de charges permanentes et $33 \%$ de charges variables :

$\mathrm{FS}=1,1 \times 1,3 \times 1,5=2,15$
$50 \%$ de charges permanentes et $50 \%$ de charges variables :

$\mathrm{FS}=1,15 \times 1,3 \times 1,5=2,24$

pieux forés, $100 \%$ de charges permanentes :

$\mathrm{FS}=1,0 \times 1,5 \times 1,5=2,25$

$67 \%$ de charges permanentes et $33 \%$ de charges variables :

$\mathrm{FS}=1,1 \times 1,5 \times 1,5=2,48$

$50 \%$ de charges permanentes et $50 \%$ de charges variables :

$\mathrm{FS}=1,15 \times 1,5 \times 1,5=2,59$

Fascicule $n^{\circ} 62$ - Titre $V$

$100 \%$ de charges permanentes :

$\mathrm{FS}=1,35 \times 1,4 \times 1,25=2,36$

$67 \%$ de charges permanentes et $33 \%$ de charges variables :

$\mathrm{FS}=1,4 \times 1,4 \times 1,25=2,45$

$50 \%$ de charges permanentes et $50 \%$ de charges variables :

$\mathrm{FS}=1,425 \times 1,4 \times 1,25=2,49$

- COMBINAISONS ACCIDENTELLES (tous les $\gamma_{\mathrm{F}}$ $=1,00$ )

Eurocode 7-1

$$
\mathrm{FS}=1,0 \times 1,0 \times 1,5=1,50
$$

Fascicule $n^{\circ} 62$ - Titre $V$

$$
\text { FS }=1,0 \times 1,2 \times 1,25=1,50
$$

On peut voir que la sécurité globale des deux règlements est très proche. En moyenne, le Fascicule $n^{\circ} 62$ - Titre V (avec les règles pressiométriques et en supposant $\xi_{\text {ave }}=1,25$ ) est légèrement plus conservatif pour les pieux foncés sous combinaisons fondamentales (jusqu'à $+16 \%$ ). Ces comparaisons devront être confirmées lorsque l'évaluation de $\xi_{\text {ave }}$ pour les règles du Fascicule $n^{\circ} 62$ - Titre $V$ sera achevée.

\section{2}

\section{Calcul sur la base d'essais de chargement statique de pieux}

\subsubsection{4}

Un cas (théorique) simple

On considère un pieu exécuté à la tarière continue (CFA) et on suppose que les résultats d'un seul essai de chargement sont disponibles, sans mesures séparées des résistances de pointe et de fût (on utile donc, tant pour l'Eurocode 7-1 que pour le Fascicule n ${ }^{\circ} 62-$ Titre $V$, le coefficient $\gamma$ ).

\section{- COMBINAISONS FONDAMENTALES et} charges permanentes seulement

Eurocode 7-1

Cas B : FS $=1,35 \times 1,0 \times 1,5=2,03$

Cas C: FS $=1,0 \times 1,4 \times 1,5=2,10$

Fascicule $n^{\circ} 62$ - Titre $V$

$$
\mathrm{FS}=1,35 \times 1,4 \times 1,2=2,27
$$

\section{- COMBINAISONS ACCIDENTELLES}

\section{Eurocode 7-1}

$$
\mathrm{FS}=1,0 \times 1,0 \times 1,5=1,50
$$




\section{Fascicule $n^{\circ} 62$ - Titre $V$}

$$
\mathrm{FS}=1,0 \times 1,2 \times 1,2=1,44
$$

Ici également la sécurité des deux règlements est très voisine, le Fascicule $n^{\circ} 62$ - Titre $V$ étant légèrement plus conservatif pour les combinaisons fondamentales (+8\%).

\subsection{2}

\section{Comparaisons sur des cas réels}

Les deux exemples qui suivent proviennent d'un projet réel pour lequel l'application des Eurocodes était demandée. Ce projet comportait plusieurs ponts et viaducs importants fondés sur pieux. Le calcul des pieux, effectué par F. Baguelin et R. Frank, en 1995, peut être résumé de la manière suivante.

\section{- PIEUX FORÉS, RÉSULTATS D'UN SEUL ESSAI DE CHARGEMENT}

a) Combinaisons fondamentales et $50 \%$ de charges permanentes et $50 \%$ de charges variables

Eurocode 7-1

Cas B : FS $=1,425 \times 1,0 \times 1,5=2,14$

Cas C: $F S=1,15 \times 1,5 \times 1,5=2,59$

Fascicule $n^{\circ} 62$ - Titre $V$

$$
\mathrm{FS}=1,425 \times 1,4 \times 1,2=2,39
$$

b) Combinaisons accidentelles

\section{Eurocode 7-1}

$$
\mathrm{FS}=1,0 \times 1,0 \times 1,5=1,50
$$

Fascicule $n^{\circ} 62$ - Titre $V$

$$
\mathrm{FS}=1,0 \times 1,2 \times 1,2=1,44
$$

- PIEUX BATTUS, RÉSULTATS DE QUATRE ESSAIS DE CHARGEMENT

a) Combinaisons fondamentales et $67 \%$ de charges permanentes et $33 \%$ de charges variables

\section{Eurocode 7-1}

Cas B : FS $=1,4 \times 1,0 \times 1,3=1,82$

Cas C: FS $=1,1 \times 1,3 \times 1,3=1,86$

Fascicule $n^{\circ} 62$ - Titre $V$

$$
\text { FS }=1,4 \times 1,4 \times 1,4=2,23
$$

b) Combinaisons accidentelles

Eurocode 7-1

$$
\mathrm{FS}=1,0 \times 1,0 \times 1,3=1,30
$$

Fascicule $n^{\circ} 62$ - Titre $V$

$$
\mathrm{FS}=1,0 \times 1,2 \times 1,14=1,37
$$

A nouveau, pour les cas réels examinés ici, les deux règlements sont en bon accord, le Fascicule $n^{\circ} 62$ Titre $\mathrm{V}$ étant plus conservatif pour les combinaisons fondamentales dans le cas des pieux battus et quatre essais de chargement $(+20 \%)$.

\section{6}

\section{Cas unique remplaçant les cas B et C}

Il apparait que, pour le problème des pieux chargés axialement, les cas B et C des ELU fondamen- taux de l'Eurocode 7-1 pourraient être remplacés par un cas unique $U$, qui intégrerait les éléments suivants

- utilisation des valeurs $\gamma_{F}$ du cas B, voir tableau I;

- utilisation pour $\gamma$, ou $\gamma_{\text {b }}$ et $\gamma_{\text {, de valeurs légèrement }}$ inférieures à celles du cas C, mais respectant pour $\gamma_{\mathrm{b}}$ et $\gamma_{1}$ la même hiérarchie (voir tableau II) :

$$
\gamma_{\text {(fonct) }} \leq \gamma_{\text {(CFA) }} \leq \gamma_{\text {(fore) }}
$$

- en conséquence, les valeurs de $\xi_{\text {ave }}$ pour le calcul à partir d'essais de sol et les valeurs de $\xi$ du tableau III pour le calcul à partir d'essais de chargement devraient être inférieures, sans doute proches de celles du Fascicule $n^{\circ} 62$ - Titre V.

Pour l'instant, nous proposons les valeurs suivantes:

$$
\gamma_{\text {tffoncel }}=1,2 ; \gamma_{\text {t(CFA) }}=1,3 \text { et } \gamma_{\text {tiforet) }}=1,4
$$

$\xi_{\text {ave }}=1,2$ pour les résultats d'essais de sol, $\xi=1,2$ quand les résultats d'un seul essai de chargement de pieu sont disponibles et $\xi=1,1$ quand les résultats de plus de deux essais de chargement de pieux sont disponibles.

L'application de ce cas unique $\mathrm{U}$ aux exemples traités ci-dessus est la suivante.

\section{1}

\section{Calcul sur la base d'essais de sol}

Pieux foncés, $100 \%$ de charges permanentes :

$\mathrm{FS}=1,35 \times 1,2 \times 1,2=1,94$

$67 \%$ de charges permanentes et $33 \%$ de charges variables :

$\mathrm{FS}=1,4 \times 1,2 \times 1,2=2,02$

$50 \%$ de charges permanentes et $50 \%$ de charges variables :

$\mathrm{FS}=1,425 \times 1,2 \times 1,2=2,05$

Pieux forés, $100 \%$ de charges permanentes :

$\mathrm{FS}=1,35 \times 1,4 \times 1,2=2,27$

$67 \%$ de charges permanentes et $33 \%$ de charges variables :

$\mathrm{FS}=1,4 \times 1,4 \times 1,2=2,35$

$50 \%$ de charges permanentes et $50 \%$ de charges variables :

$\mathrm{FS}=1,425 \times 1,4 \times 1,2=2,39$

Ces résultats sont, dans la plupart des cas, très proches de ceux donnés par les relations (8) à (16) et ne diffèrent jamais de plus de $10 \%$. Incidemment, les règles pressiométriques du Fascicule $n^{\circ} 62$ - Titre V (relations 17 à 19) sont maintenant plus conservatrices, en moyenne, dans tous les cas de combinaisons fondamentales $(+22 \%$ pour les pieux foncés et $+4 \%$ pour les pieux forés).

\section{2}

\section{Calcul sur la base d'essais de chargement de pieux}

Exemple théorique : pieu CFA, un seul essai de chargement de pieu :

$$
\mathrm{FS}=1,35 \times 1,3 \times 1,2=2,11
$$

Ce résultat doit être comparé à ceux des relations (22) et (23). Il est tout à fait satisfaisant. Le Fascicule 
$n^{\circ} 62$ - Titre V (relation 24) est plus conservatif de $8 \%$.

Cas réel : pieux forés, un seul essai de chargement, $50 \%$ de charges permanentes et $50 \%$ de charges variables:

$$
\mathrm{FS}=1,425 \times 1,4 \times 1,2=2,39
$$

Ce résultat doit être comparé à ceux des relations (27) et (28). Il est tout à fait satisfaisant. II est exactement le même que pour le Fascicule $n^{\circ} 62$ - Titre V (relation 29).

Cas réel : pieux battus, quatre essais de chargement, $67 \%$ de charges permanentes et $33 \%$ de charges variables :

$$
\mathrm{FS}=1,4 \times 1,2 \times 1,1=1,85
$$

Ce résultat doit ètre comparé à ceux des relations (32) et (33). Il est tout à fait satisfaisant, Le Fascicule $n^{\circ} 62$ - Titre $V$ (relation 34 ) reste plus conservatif d'environ $20 \%$.

\section{Conclusion}

Bien que les approches puissent paraitre différentes et qu'il y ait des différences dans les valeurs des coefficients de sécurité partiels, les sécurités globales de la prénorme européenne Eurocode 7-1 et du règlement officiel français Fascicule $n^{\circ} 62$ - Titre $V$ sont très comparables, lorsque l'on dimensionne des pieux sous charges axiales vis-à-vis de la capacité portante ultime.

Les règles de calcul à partir des résultats d'essais in situ (pressiométriques et pénétrométriques) données dans le Fascicule $n^{\circ} 62$ - Titre V ont besoin d'être comparées aux résultats des essais de chargement de pieux qui ont permis de les constituer, afin de vérifier leur compatibilité avec les exigences de l'Eurocode 7-1.

Pour le problème de la capacité portante des pieux chargés axialement, un cas unique pour les combinaisons fondamentales ELU, remplaçant les cas B et C de I'Eurocode 7-1, peut aisément être trouvé.

\section{Bibliographie}

AFNOR-Eurocode 7. Calcul géotechnique, Partie 1: Règles générales. XP ENV 1997-1 (P 94-250-1), Paris, Association Française de Normalisation (AFNOR), 112 p., 1996.

Bustamante M.. Frank R. - Desian of axially loaded piles in France : National Report. Proc. Int. Seminar Design of axially loaded piles, European practice, ERTC3, Brussels. F. de Cock \& C. Legrand, eds, Rotterdam, Balkema, p. $161-175,1997$.

Bustamante M.. Gianeselli L. - Prévision de la capacité portante des pieux isolés sous charge verticale, Règles pressio- métriques et pénétrométriques. Bulletin de liaison des LPC, $\mathrm{n}^{\circ} 113$, p. 83-108, 1981.

CEN-ENV 1997-1 Eurocode 7, Geotechnical Design, Part 1: General Rules. Comité Européen de Normalisation (CEN). TC 250/SC7, Bruxelles, 123 p., 1994.

Frank R, - The new Eurocode and the new French code for the design of deep foundations. Keynote lecture. Proc. FHWA Int. Conf. Design and Construction of Deep Foundations, Orlando, Florida, vol. 1, Washington, D.C., FHWA. p. 279-304, 1994.
Frank R. - Some comparaisons of safety for axially loaded piles. Proc. Int. Seminar Design of Axially loaded piles, European Practice, ERTC3, Brussels, F. de Cock \& C. Legrand, eds, Rotterdam, Balkema, p. 39-46, 1997.

ISSMFE - Subcommittee on Field and Laboratory Testing. Axial plle loading test, Part 1: Static loading. Geotechnical Testing J., 8 (2), p. 79-90, 1985.

MELT - Règles techniques de conception et de calcul des fondations des ouvrages de génie civil . CCTG Fascicule $n^{\circ} 62$ Titre V. Paris, ministère de l'Équipement du Logement et des Transports, 1993. 